\title{
Epistemología, complejidad y competencias ${ }^{1}$
}

José Humberto Flores $M^{2}$

"Es necesario que el cuerpo docente se sitúe

en los lugares más avanzados del peligro

que constituye la incertidumbre permanente del mundo" (Martin Heidegger)².

\section{Resumen}

Para introducir los temas de la teoría del conocimiento, la epistemología, la ciencia, la verdad y la filosofía nos parecen sugerentes las palabras que escribía san Agustín en De Trinitate IX, 1) en el siglo IV de nuestra era:

"Busquemos como buscan los que aún no han encontrado, y encontremos como encuentran los que aún han de buscar, porque cuando el hombre ha terminado algo no ha hecho sino comenzar".

\section{Introducción}

$\mathbf{N}$ o hay duda de que los conceptos de teoría del conocimiento o la epistemología tienen su base en el concepto griego de filosofía. Este concepto de filosofía, desde el principio, no significaba únicamente la posesión del conocimiento, sino, y sobre todo, la búsqueda del conocimiento. Zubiri, siguiendo a Husserl y a Heidegger, decía que la filosofía "ha sido ante todo una justificación o un esfuerzo mostratorio de la existencia (sit venia verbo) de su objeto", que se une al concepto aristotélico que afirmaba que la filosofía es "el saber que se busca" (Zetoumene episteme) (Zubiri, 2009:14).

Este saber que se busca no se realiza solo sobre el método, sino, además, sobre el objeto mismo de la filosofía. En otras palabras, comprenderemos que la filosofía está en un permanente dinamismo y en permanente búsqueda de sí misma; y, sobre todo, en permanente búsqueda de su objeto de estudio. En su Metafísica, Aristóteles menciona que los modos del saber, donde se patentiza la verdad como $\alpha \lambda \varepsilon \tau \theta \iota \alpha$, como desvelamiento de las cosas, son la techné, la phrone-

1 Conferencia dictada el sábado 1 de noviembre a los estudiantes de la Maestría en Docencia e Investigación Educativa.

2 Doctor en Filosofía y licenciado en Teología y en Filosofía.

3 La cita del filósofo alemán, Martin Heidegger, en: Morin, E. (2000). La mente bien ordenada. Barcelona: Editorial Seix Barral. 
sis, la episteme, el nous y la sophía. Así, la episteme que es la demostración de las cosas, que significa un verdadero saber de las cosas, se une al Nous, que se comprende como conocimiento apodíctico que se constituirá al final en la sophia que significa el saber verdadero.

La búsqueda del saber y del conocimiento verdadero fueron los pilares de la teoría del conocimiento, ocupándose de los problemas del origen, la posibilidad y la esencia del conocimiento (Hessen, 1981). Sin embargo, en las últimas décadas, la cuestión de la teoría del conocimiento se estudia como epistemología. La epistemología, como la episteme en los griegos, se concebía como 'la pretensión de alcanzar conocimientos válidos', explícito en la idea de ciencia como 'búsqueda humana y racional, en la que el hombre encuentra criterios fiables para avanzar en el desarrollo de diversas prácticas' (Vargas, 2006:39).

En otras palabras, podemos decir que el mundo griego concebía la epistemologìa de modo distinto a como se concibe en el mundo actual, donde encontramos modos de conocimientos verdaderos o epistemias (Foucault, 1985:78). La epistemología, tal como la conocemos ahora, es considerada como la disciplina que estudia la producción de conocimientos y las articulaciones que existen entre distintas disciplinas, y que se pregunta cuál es el objeto de la investigación, cómo adquirir los conocimientos o cómo una ciencia puede tener una pertinencia social. La epistemología, por tanto, es determinante en la educación superior, y, sobre todo, en la acción educativa y en la investigación.

Las siguientes líneas pretenden establecer la epistemología que subyace en el modelo por competencias desde el pensamiento complejo. En otras palabras, trata de dar razones de por qué es necesario prestar atención al aprendizaje sistémico, donde los estudiantes son los sujetos de ese aprendizaje, capaces en la toma de decisiones que hacen en los diferentes contextos que les toca enfrentar. La complejidad será abordada por la importancia que esta tiene en su carácter sistémico y amplio, y, sobre todo, porque tratará problemas no solo sociales, sino, y sobre todo, éticos y ambientales.

\section{El concepto de competencias}

Existe una gran variedad de conceptos y definiciones que captan la naturaleza de la competencia. Muchas veces estos conceptos se visualizan únicamente desde la óptica laboral y práctica. Sin embargo, estamos persuadidos de que el concepto de competencias se traduce en algo más amplio y extendido. Este concepto proviene del latín competeré, que está vinculado con 'pertenecer a algo', con 'incumbencia', o bien, con la 'pericia de hacer algo'. En un sentido más amplio, se puede entender como 'la aptitud para hacer algo'. Con ello nos alejamos de las concepciones funcionalistas ${ }^{4} \mathrm{y}$ conductistas ${ }^{5}$ de competencias, y apuntamos a una conceptualización más holística e integral de estas. Para tal situación, nos colocamos en tres dimensiones que interactúan entre sí: las actuaciones, las competencias y la complejidad. El concepto puede definirse como: "Saber actuar de manera pertinente en contextos complejos e inciertos ${ }_{\iota}$ enfrentando con claros criterios de calidad aquellos problemas que le son propios o apropiados a la profesión, para lo cual se seleccionan y movilizan recursos personales (conocimientos, actitudes, valores, experiencia), de redes (de expertos, de información) y del contexto, en orden a resolverlos, estando en condiciones de dar razón (científica, tecnológica, política, económica, ética) de sus decisiones y haciéndose cargo de la misma" (adaptado de Troncoso \& Hawes, 2007).

\footnotetext{
4 En esta visión se asumen las competencias como atributos que deben tener las personas para cumplir con los propósitos de los procesos laborales-profesionales desde unas funciones definidas.

5 La conductual asume las competencias como comportamientos clave de las personas para la competitividad en las instituciones.
} 


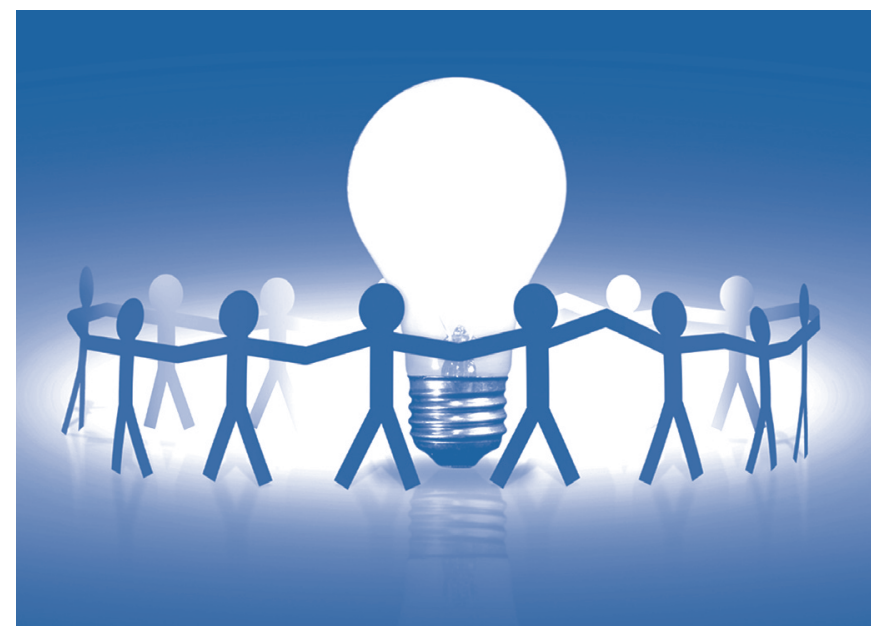

Este saber actuar, sin embargo, no solo estará en relación con los saberes que expuso Delors (1997) hace unos años, sino que se pondrá en un contexto en el cual los estudiantes tendrán la oportunidad de interactuar con lo complejo de la realidad. Así, el saber actuar estará orientado a varias facetas de la vida, tales como el saber hacer del mundo laboral; el saber aprender en su desarrollo profesional; el aprender a convivir de su entorno social; y el aprender a ser, en su vida misma.

El saber actuar en general responderá, a la vida misma, de manera holística e integral donde entran en acción todas las dimensiones del ser humano: ${ }^{6}$ la inteligencia, la voluntad, el sentimiento, etc. Con el saber actuar la necesidad de aprender a vivir cobra nuevas dimensiones, y capacita al ser humano para afrontar contextos complejos e inciertos. El aprendizaje, por tanto, se debe reforzar desde la incertidumbre. Es necesario distanciarse del recetario para la solución de los problemas. Durkheim (1890:38) comentaba, al referirse a la enseñanza del estudiante: se debe "crear en él un estado interior y profundo, una especie de polaridad del alma que le oriente en un sentido definido no solo durante la infancia, sino para la vida entera".
No se trata, pues, de transmitir y de llenar de datos las mentes de los estudiantes, sino que se trata de crear una habitud en ellos para que aprendan a actuar en contextos complejos que van más allá de su disciplina de estudio y para que aborden aspectos de la vida misma.

Este saber actuar ante los contextos complejos nos lleva a movilizar nuestros conocimientos y competencias a fin de tomar buenas decisiones para la resolución de problemas. Esta movilización de los recursos personales hace posible relacionar el saber, construir conocimiento, adquirir nuevas competencias, con el objetivo de estar mejor equipados ante los desafíos del contexto. En síntesis, conocemos datos, evaluamos situaciones, etc., para la toma de decisiones en los diferentes ámbitos de la vida, como el académico, el social, el laboral, etc.

\section{Las competencias desde el pensamiento complejo}

En la actualidad existe una crítica al hecho de creer que solamente existe un solo estatuto científico válido. Dígase lo mismo del reconocimiento de una epistemología que conduce a una visión de ciencia que se centra en la ciencia positiva. No es extraño que en esta situación se ponga en crisis una epistemología centrada en los criterios de demarcación (Candioti, 2009:151). En este sentido, la racionalidad ya no puede ser el único fundamento de la ciencia, sino que se debe ir más allá de esta visión, reconociendo que hay otras formas de entender la ciencia y la epistemología, que se habían reclamado como únicas visiones de entender al sujeto en su relación con el mundo. Esta nueva visión ha permitido una ruptura en la visión de la ciencia, proclamando en unos casos la discontinuidad de ella misma (Kuhn, 1971) ${ }^{7}$ y

\footnotetext{
6 Esta visión holística e integral más cercana al pensamiento complejo nos parece más pertinente que el enfoque constructivista, ya que este tiene la base en la construcción del conocimiento a partir de la razón, dejando a un lado la voluntad, el sentimiento, etc., y resuelve dificultades en los procesos laborales y profesionales desde un punto de vista organizacional, dejando a un lado la posición ética, la calidad de vida, el desarrollo social y el equilibrio con el ambiente.

7 Kuhn, al igual que Feyerabend, apelan a la discontinuidad y a las rupturas en el conocimiento científico ya que este apela a las diversas formas de vida, a la variedad de los juegos lingüísticos y a los marcos paradigmáticos. Para ellos la epistemología de la demarcación solo obedece a unos criterios y no da cuenta de la praxis en lo social.
} 


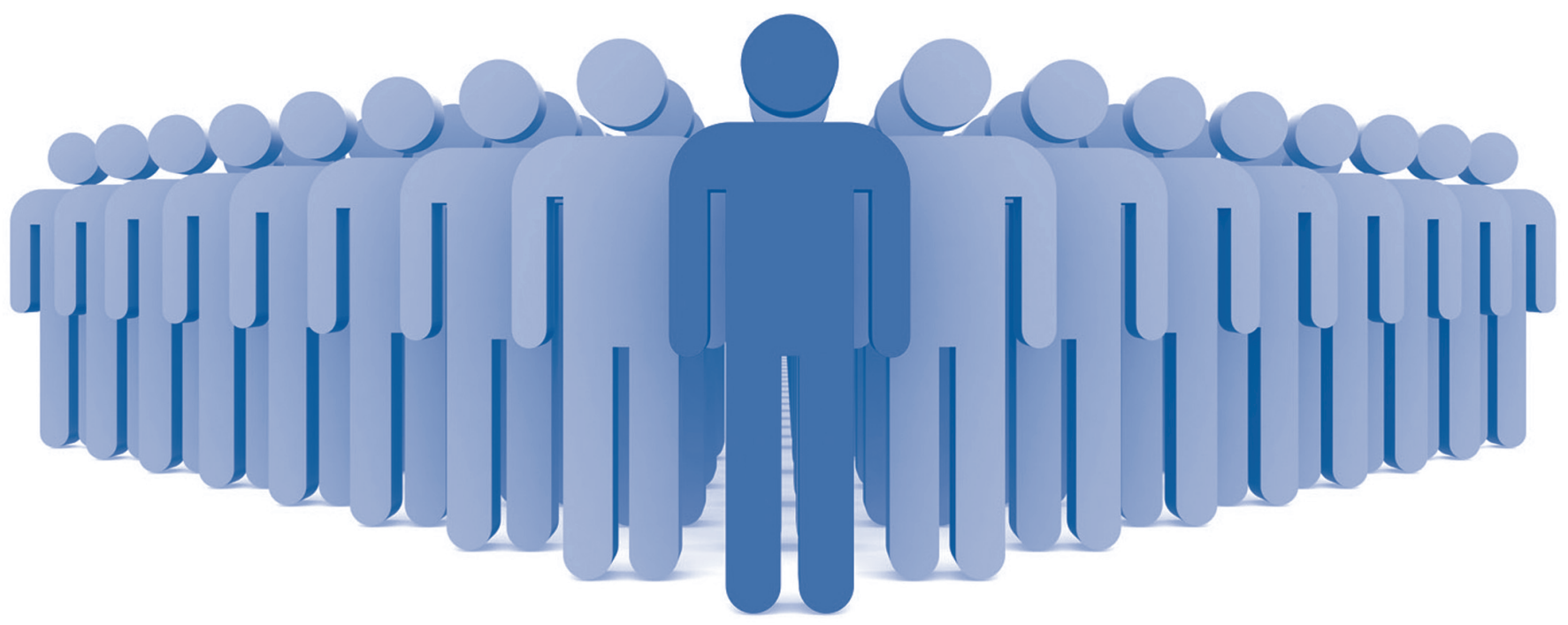

en otros casos, la anarquía en la visión científica prevalente (Lakatos, 1983). ${ }^{8}$ Aún cuando no pretendemos seguir el camino radical de una superación de la epistemología clásica, al estilo de Rorty (1989 y 1997), ${ }^{9}$ sí podemos plantearnos un nuevo modo de reflexión en este tema.

Ciertamente no podremos hablar de un solo enfoque epistemológico y, peor aún, de un enfoque centrado en el positivismo que antes hemos expuesto.

Para considerar nuevos caminos para la epistemología, ha sucedido como acto anterior el reconocimiento de que también ha habido nuevos caminos de comprensión de la racionalidad. Para ello han colaborado "las concepciones hermenéuticas, las ideas de Wittgenstein acerca de los juegos lingüísticos, la Teoría de la Acción Comunicativa de Habermas y los estudios semióticos de Apel. Éstos nos han mostrado no solo un modo diferente acerca del lenguaje, sino la ruptura de los presupuestos de la epistemología moderna y, en definitiva, una concepción diferente de la racionalidad, que no puede concebirse ya como una realidad epistémica, sin considerar su carácter comunicativo-discursivo" (Candioti, 2009:176).

Esta consideración comunicativa de la epistemología nos abre el horizonte para pensar que no nos centraremos en una ciencia única y autista, sino que nos abriremos al concurso de otros enfoques epistémicos que nos hacen posible la interdisciplinariedad. Esta referencia de las diferentes disciplinas necesitará, sin duda, un carácter dialógico, comunicativo e intersubjetivo capaz de salir de su propia órbita de especialización para confrontarse con otros saberes necesarios para el acercamiento a la verdad. En esta nueva visión epistémica consideraremos que el conocimiento no

8 Lakatos también rompe con los criterios de demarcación de la ciencia positiva, y él propone una epistemología criterial que obedezca a situaciones del contexto y de la historia. Estos criterios no serán inmutables (como sucede en el cientismo positivista), sino que son contingentes que podrán variar según la necesidad del contexto.

9 Richard Rorty, filósofo norteamericano que propuso el giro pragmático no solo de la ciencia, sino también de las prácticas sociales. Para Rorty es necesario la ruptura entre la racionalidad y las prácticas sociales. Cuando el ser humano practica la solidaridad lo hace ante la necesidad del necesitado, y no actúa porque detrás del acto solidario haya una fundamentación o una racionalidad que le indique el camino correcto. El rechazo de la epistemología clásica la hace Rorty porque considera que la construcción epistemológica positivista y moderna también es fruto de una racionalidad sospechosa anclada en los grandes relatos de la modernidad. 
es la representación de una realidad ya dada con antelación, sino que es un proceso de construcción significativa, en una estrecha relación con las actividades sociales.

Desde esta visión epistémica, necesariamente nuestras prácticas comunitarias y sociales tienen que cambiar: se harán más significativas porque nos llevan a afrontar permanentemente a contextos ${ }^{10}$ complejos de la realidad. En síntesis, necesitamos de epistemologías, de formas plurales de comprender la realidad, ya que ella es compleja en sí misma. Atenernos a una sola disciplina para el conocimiento de la realidad nos llevará irremediablemente a ver solo una parte de ella y de forma parcializada. Y en el caso de las comunicaciones se hace exigible la atención a otras disciplinas, ya que ella de suyo sobresale por su carácter dialógico, comunicativo e interactivo. La educación, por tanto, no puede vivir en el autismo que las ciencias positivas vivieron en el pasado.

Dado lo anteriormente dicho, es necesario que ante contextos complejos nos reubiquemos epistemológicamente (Morin, 2005:27). Como hemos visto, no seguimos una episteme rígida que constituía una forma unilateral de hacer ciencia, tal como lo afirmaba el discurso positivista. Es claro que no vamos detrás de la búsqueda de una ciencia que imponga leyes y que simplemente legisle. El caso de las comunicaciones es distinto. La epistemología que ahora nos planteamos es aquella en la cual nos preguntamos ¿cómo conozco?, ¿cómo me sitúo en la realidad?, ¿cómo aprendo? y ¿cómo me comunico? Por ejemplo, en la globalización, que de suyo es un contexto complejo, se requiere de toda esta serie de preguntas que nos acabamos de formular. Esto sin duda nos ha llevado a una nueva forma de aprender, que nos ayuda a afrontar la educación desde un mundo complejo, globalizado y comunicado. No basta, entonces, un cambio de métodos o de planes de estudio, sino que se impone un cambio en el pensamiento y en la elaboración de nuestras construcciones mentales.

Pero esta nueva manera de abordar el conocimiento nos permite superar las antiguas antonomias, como la división entre teoría y práctica; la diferencia entre ciencias duras y ciencias blandas o la diferenciación de diferentes disciplinas. Así, "Ias ciencias de la complejidad instauran efectivamente una nueva forma de racionalidad, distinta a las conocidas en la historia de Occidente, y en la que no caben ya oposiciones entre los planos teórico y práctico, o filosófico y científico, o teórico y social, que fueron los que marcaron al grueso de la historia de la ciencia tanto como de la historia de la filosofía" (Maldonado 2003:153).

Con esta visión del problema nos encontramos con que la adopción de la perspectiva de complejidad no se hace solamente por una moda: se trata de un cambio epocal que ha afectado, sin duda, el campo de la epistemología. La característica de este cambio epocal ha consistido en redefinir las raíces de las diferentes disciplinas. ${ }^{11}$ En este contexto, la ciencia ya no solamente tratará sobre la descripción de leyes naturales y la explicación de fenómenos, sino que la ciencia y el aprendizaje se dirigen hacia toda la vida, afectando el ethos de los ciudadanos.

El pensamiento complejo sale al paso no solamente de soluciones simples, sino que se opone a la megaespecialización de los profesionales, ya que es la renuncia a afrontar los problemas de manera integral. Con la especialización solamente se observa una faz del problema. Los expertos dicen que se tiene que ir más allá de un pensamiento especializado, así como lo cita Hawes (2006): "Actualmente el capital del

10 No debemos caer necesariamente al contextualismo de Rorty, el cual afirmaba el divorcio de nuestros actos con condicionamientos de racionalidad previamente establecidos. En este sentido, los seres humanos actuarán según el contexto que lo determine.

11 Este giro epocal, que afecta a la epistemología, viene dado en parte por la reflexión y la crítica postmoderna ha hecho en contra de la razón ilustrada de la modernidad que predominó durante los siglos XIX y XX en el campo de la educación. 


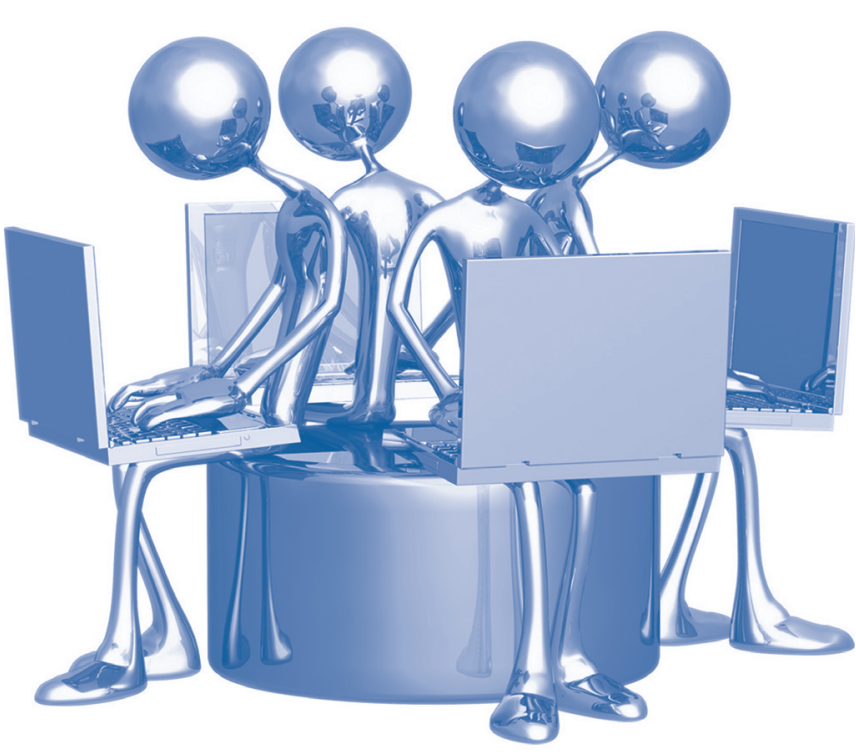

conocimiento instalado en las distintas disciplinas se multiplica por dos cada cinco años. Esto es lo que hace absurdo formar profesionales muy especializados en áreas específicas, porque a mayor especialización mayor es la caducidad del conocimiento. La tendencia es que el profesional tenga ahora el know why, esto es, que sea capaz de explicarse cómo ocurren las cosas; el know what, esto es, qué ocurre en su dimensión más descriptiva; el know how, que es una competencia asociada a las prácticas, y el know who, puesto que el conocimiento está en redes, y lo importante para el profesional es saber quién lo tiene y dónde está" (Moeller \& Rapoport, 2003). ${ }^{12}$

El estudiante, por tanto, debe aprender a actuar en contextos complejos más allá de su propia disciplina de especialización. Debe aprender a buscar el complex, ${ }^{13}$ es decir, aprender a relacionar todos los saberes y todo lo que está por saber.

El pensamiento complejo, por tanto, nos ayuda a unir, reunir, construir y relacionar los conocimientos, esos conocimien- tos que están en constante cambio. Este pensamiento tiene ciertas características (Morin, 1994 y 2001), a saber: el principio dialógico donde los principios antagónicos se unen sin perder sus diferenciaciones; la recursión organizacional, en donde los procesos se autoreproducen y autoorganizan en tanto los efectos producen causas y las causas, efectos; el principio hologramático, en el cual el sistema todo está en la parte, y la parte está en el todo; reconoce el continuo proceso de interacciones entre partes; combina lo cuantitativo con lo cualitativo; y considera la realidad como un proceso en continuo cambio, entre otros.

En este contexto en que se diversifican las epistemologías es necesario reconcebir los saberes hacia una mirada desde la complejidad (Morin, 1991, 1994). En este sentido, los profesionales de cualquier disciplina deben actuar articulando y movilizando todos los saberes, incluso de aquello de lo que no se sabe. En esta articulación de saberes el profesional podrá percibir las demandas de nuevos saberes que le plantean los problemas. En la complejidad resulta necesario tener la mirada en el exterior donde las profesiones tienen su máxima referencia. También es necesario tener la mirada en el interior para profundizar en la pregunta universitaria: ¿Qué saberes estamos enseñando y aprendiendo? ¿Los saberes están en una relación dialógica con las profesiones? ¿La relación entre el saber y las profesiones superan la vieja antinomia entre la teoría y la práctica?

Toda esta visión recoge aspectos integrales como la actuación, la idoneidad, la flexibilidad y el desempeño generalizado que nos ayudará a ilustrar las competencias desde la complejidad, como lo afirma Tobón (2007): "Procesos complejos de desempeño con idoneidad en determinados contextos, integrando diferentes saberes, para realizar actividades y/o resolver problemas con sentido de reto, motivación, flexibilidad, creatividad, comprensión, emprendimiento...".

\footnotetext{
12 Sin embargo, estas competencias deben ser llevadas a procesos más complejos donde se denote las actuaciones, la resolución de problemas con idoneidad y ética, la calidad de vida, el equilibrio con el ambiente, etc.

13 La complejidad proviene etimológicamente de la palabra en latín complex que significa "lo que está tejido junto."
} 


\section{La centralidad en el aprendizaje contextualizado}

Desde hace unos años atrás, el acto educativo señala como prioritario el aprendizaje sobre la enseñanza. Aunque es cierto que no se puede dejar de enseñar, también es cierto que se privilegia el aula, el ambiente universitario, las investigaciones, las revistas científicas, la educación a distancia, etc., como lugares excepcionales para el aprendizaje. Es más: Ios actores del aprendizaje somos todos. Y estas certezas que tenemos del aprendizaje también ha permeado las bases epistemológicas de las diferentes disciplinas. Es más recurrente preguntarnos ahora ¿cómo conozco?, ¿cómo aprendo mejor?, etc.

Y en estas valoraciones del aprendizaje contextualizado tenemos dos predecesores que han influido en el campo de la educación, sobre todo, a escala latinoamericana. El primero fue Jean Piaget, quien propuso el paradigma cognitivo donde el sujeto cognoscente construye su propio conocimiento en referencia con el contexto que le toca vivir. Así, según Piaget, la educación consistirá en "crear hombres que sean capaces de hacer cosas nuevas, no necesariamente repetir lo que han hecho otras generaciones: hombres que sean creativos, inventivos y descubridores. El segundo aspecto de la educación es formar mentes que puedan criticar, que puedan verificar y no aceptar todo lo que se les ofrezca" (Hernández, 1998:192). En esta visión el sujeto-estudiante es un constructor activo de su conocimiento desde su contexto. Con todo lo positivo que ha sido este enfoque nos damos cuenta de que el aprendizaje se encierra únicamente en el individuo y que su referencia al contexto es secundaria.

El otro referente en el campo educativo ha sido Vigotsky, que propuso el paradigma sociocultural con el cual trataba de superar los estudios fragmentados de los procesos psicoló- gicos superiores que no tenían una relación entre sí. Suponía que el aprendizaje es un proceso estrictamente social, a través del cual se vinculan las herramientas y los signos culturales. Así, las metas educativas se construirán en función de lo que la cultura considera valioso, entendidos los estudiantes y los docentes como seres sociales productos de interacciones socioculturales de las que son parte (Rodríguez, 2009:104). Aún cuando se visualiza que es un modelo más contextualizado, el aprendizaje siempre gira en la órbita del sujeto, y muchas veces ese aprendizaje no regresa en productos al ámbito social y cultural.

En el pensamiento complejo se privilegian las actuaciones del sujeto con idoneidad y ética, buscando su realización personal y calidad de vida, en franca apertura al desarroIlo social y en equilibrio con el ambiente. La complejidad, a diferencia del constructivismo cognitivo y el socioconstructivismo, hace que el individuo coloque sus conocimientos y competencias en vista de la construcción de un ethos que impacta significativamente en la vida social, económica y medioambiental de los miembros de la comunidad

La complejidad se traducirá hasta el campo del aprendizaje. Haciendo un modelaje de la complejidad en la vida universitaria, podemos poner el ejemplo del método de casos, ya que permite a los estudiantes situarse en un plano de la realidad de forma sistémica e interdisciplinaria. Ayuda, también, a que se experimenten varios métodos y prácticas sociales en los cuales el conocimiento, las preguntas, las soluciones y el aprendizaje se hagan en forma dinámica e interactiva, logrando que ellos puedan constituir un aprender a aprender de forma original.

El método de casos ayudará a situaciones decisivas del aprendizaje. Presentamos aquí brevemente, algunos elementos que tener en cuenta. 
A) Puede dar relevancia a los datos que tenemos, ya que a veces no sabemos qué hacer con lo que sabemos. B) En las intervenciones educativas, para que se dé un buen aprendizaje, se deben privilegiar los caracteres dialógicos e interactivos de la educación. C) Para estudiar lo complejo es necesario trabajar con fuentes distintas y de distinto enfoque epistemológico. Cuanto más sepamos mejor comprenderemos, podremos hacer mejores previsiones y, por tanto, comportarnos en consecuencia. Esto se acentúará si los conocimientos pertenecen a áreas distintas. D) No se podrá separar el conocimiento sobre algo o alguien de los contextos e interacciones que los afecten. E) El estudio de un caso con profundidad ayuda a conocer mejor otros casos, por más que éstos sean distintos. Por ejemplo, entendemos mejor a un pintor si ya conocemos mejor a otros pintores. F) Cuando estudiamos un caso en profundidad, el conocimiento que obtenemos de él nos afecta también a nosotros (Aznárez \& Callejón, 2006:189-190). El método de casos nos ayuda a privilegiar el aprendizaje en contextos complejos e inciertos, a prepararnos para tomar las mejores decisiones a partir de la movilización de nuestros recursos y los de los demás.

No hay duda de que el docente y el investigador son protagonistas de este cambio epistemológico y curricular. Este giro no solo significará el cambio de mentalidad del docente, sino que él mismo, desde su disciplina científica, ayudará cambiar su universidad.

En la epistemología de las disciplinas se conjugan algunos elementos determinantes para determinar la cientificidad de una disciplina. Estos son: el objeto, el método, la validez, la historicidad y la enseñabilidad de la ciencia (Vargas, 2006:19). En un proyecto de innovación curricular, los docentes pondrán la especificidad de sus disciplinas como contribución académica. Las razones de este hecho descansan en que, para conjugar todo al enfoque de competencias, las disciplinas deben girar con el objeto, el método, la validez y la historicidad de su ciencia. El cambio de las competencias, por su parte, se centrará en el último elemento de la epistemología de la disciplina: la enseñabilidad. En la enseñabilidad, que en un sentido amplio denota el aprendizaje, la investigación y la transmisión de conocimientos y de competencias, se desplegará el enfoque por competencias. Para este cometido, el docente confrontará la didáctica y la evaluación en la enseñabilidad de su disciplina. Las didácticas y las evaluaciones deben ser dirigidas hacia la capacidad de actuación en situaciones complejas.

Si el paradigma tradicional se basaba en establecer las bases del pensamiento lógico donde giraba su atención en las operaciones del pensamiento, como observar, describir, comparar y razonar; ahora el paradigma actual entreve no solo en lo anterior, sino en un pensamiento tecnológico que nos da la capacidad para actuar en la realidad y tomar las mejores decisiones. Ciertamente, no se trata de derribar el pensamiento lógico, ya que este es necesario; sin embargo, este pensamiento lógico debe redefinirse en el pensamiento tecnológico, donde se ponen en acto competencias de acción que son necesarias para el mundo laboral, la vida ciudadana y la vida personal (Aguerrondo,2009:9).

Para actuar en la realidad los estudiantes necesitarán un pensamiento sistémico (systems thinking) ${ }^{14}$ que no solo resolverá las causas de los problemas, sino que, además, permitirá un horizonte más amplio de los saberes, una visión holística de las cosas, una observación de las relaciones dinámicas en redes (en lugar de buscar cadenas lineales de causa-efecto), el reemplazo de la consideración unidimensional de las cosas por planteamientos pluridimensionales, la sustitución de Ios pensamientos deterministas por planteamientos abiertos

\footnotetext{
14 Es importante la contribución de Senge en su obra La quinta disciplina, en Senge, P. (1990). The Fifth Discipline. Londres. Publicado en español: La quinta disciplina. Barcelona: Granica).
} 
que posibiliten la innovación, privilegiar las competencias en el manejo de la información, de los medios y tecnologías de la información y la comunicación (TIC) y, en suma, actuar con habilidades para la vida personal y profesional.

Si la centralidad en este modelo de competencias recae en el estudiante, entonces la educación a distancia será una herramienta tecnológica necesaria para el estudiante al momento de enfrentarse a contextos complejos. Esta herramienta tecnológica no solamente supone manejo de las TIC, sino que, además, necesitará métodos, construcciones teóricas y prácticas, didácticas y evaluaciones que hagan posible adentrarse en esta nueva forma de aprendizaje continuo. Ciertamente, las TIC y la tecnología de la educación a distancia hacen que el aprendizaje sea continuo y dinámico, desafiando permanentemente a estudiantes y profesores a mejorar en sus saberes y sus profesiones.

\section{REFERENCIAS}

- Aguerrondo, I. (2009). "Conocimiento complejo y competencias educativas." IBE Working papers on Curriculum Issues, № 8 , Ginebra: Unesco- IBE.

- Aznárez, J.P. y Callejón, M.D. (2006). "La necesidad de trabajar con procesos de conocimiento y comprensión complejos." SeviIla: Escuela Abierta.

- Durkheim, E. (1890). L'Evolution pédagogique en France. Paris: PUF, en, Morin, E. (2000). La mente bien ordenada. Barcelona: Editorial Seix Barral.

- Foucault, M. (1985). Las palabras y las cosas. México: Siglo XXI.

- Gardner, H. (2001). Las inteligencias múltiples hacia el siglo XXI. Barcelona: Piadós.

- Hernández, G. (1998). Paradigmas en Psicología de la Educación. México: Paidós.

- Hessen, J. (1981). Teoría del conocimiento. México: Editores Mexicanos Unidos.

- Kuhn, T. (1971). La estructura de las revoluciones cientificas. México: FCE.

- Lakatos, I. (1983). La metodología de los programas de investigación científica. Madrid: Alianza Editorial.
Maldonado, C.E. (2003). "Marco teórico del trabajo en ciencias de la complejidad y siete tesis sobre la complejidad." En Revista Colombiana de Filosofía de la Ciencia (Universidad del Bosque, Bogotá) Vol. 4, № 8-9.

Morin, E. (1991). "La necesidad de un pensamiento complejo." En S. González Moena (Ed.), Pensamiento complejo. En torno a Edgar Morin, América Latina y los procesos educativos. Santafé de Bogotá: Magisterio.

Morin, E. (1994). Introducción al pensamiento complejo. Barcelona: Gedisa.

- Morin, E. (2000). La mente bien ordenada. Barcelona: Editorial Seix Barral.

- Moeller, P. y Rapoport, D. (2003). "Observatorio del empleo," en, Corvalán, O. y Hawes, G. (2006), Aplicación del enfoque de competencias en la construcción curricular de la Universidad de Talca, Chile. Talca: Revista Iberoamericana. Ver detalles en http:// www.utalca.cl/mecesup2/html/proyecto_tal0101/pag1.htm.

Pedroza, R. (2005). “La flexibilidad académica en la universidad pública". En Flexibilidad académica y curricular en las instituciones de educación superior, pp. 19-41. México: Porrúa.

- Piaget, J. (2003). La psicología de la inteligencia. Barcelona: Crítica.

- Rodríguez, M. (2009). "Educación para la transformación.” En Sophia, Subjetividad: consideraciones desde la filosofía de la Educación. Quito: Editorial Universitaria Abya-Yala, № 7, pp. 93-118.

- Rorty, R. (1989). La filosofía y el espejo de la naturaleza. Madrid: Cátedra.

- Rorty, R. (1997). ¿Esperanza o conocimiento? Una introducción al pragmatismo. Buenos Aires: FCE.

- Tobón, S. (2007). "El enfoque complejo de competencias y el diseño curricular por ciclos propedéuticos". En Acción pedagógica 16: $14-28$

- Troncoso, K. \& Hawes, G. (2007). "Conversaciones académicas en torno al curriculum basado en competencias. Primera conversación" [Presentación Power Point]. Santiago: Universidad de Chile, Vicerrectoría de Asuntos Académicos.

- Vargas, G. (2006). Filosofía, Pedagogía, Tecnología. Bogotá: San Pablo.

Zubiri, X. (2009). Cinco lecciones de filosofía. Con un nuevo curso inédito. Madrid: Alianza Editorial. Fundación Xavier Zubiri. 